\title{
Enhancement of Androgen Action in the Kidneys of Transgenic Mice Harboring the Mutant Human UMOD Gene
}

\author{
Yuichi Takiue $^{1}$, Makoto Hosoyamada ${ }^{1, *}$, Masaki Kimura ${ }^{1}$, and Hidetsugu Saito ${ }^{1}$ \\ ${ }^{1}$ Division of Pharmacotherapeutics, Keio University Faculty of Pharmacy, \\ 1-5-30 Shibakoen, Minato-ku, Tokyo 105-8512, Japan
}

Received September 15, 2010; Accepted January 17, 2011

\begin{abstract}
Uromodulin storage diseases are characterized by hyperuricemia of underexcretion type and renal insufficiency. Although these diseases are caused by mutations in the UMOD gene that encodes the kidney-specific glycoprotein uromodulin, the effect of uromodulin mutation on the kidney has not been clearly established. In this study, we investigated the effect by comparing transgenic mice expressing human uromodulin with and without mutation. Change in the intracellular localization of human uromodulin protein was shown in the kidney of transgenic mice expressing mutant human uromodulin by a deglycosylation experiment. Then, we determined by microarray technology and quantitative real-time PCR that the strongly induced gene in the kidney of these mice was 5 - $\alpha$-reductase 2 , an enzyme that converts testosterone into the more potent androgen. Moreover, the expressions of androgen-induced genes $\beta$-glucuronidase, ornithine decarboxylase structural 1, and cytochrome P450 4a12a were increased. The increase in mRNA levels of urate reabsorptive transport system urate transporter 1 could be investigated, but the changes in its protein level and renal urate handling could not be demonstrated. Therefore, it is suggested that a uromodulin mutation may be responsible for the enhancement of renal androgen action.
\end{abstract}

[Supplementary Table: available only at http://dx.doi.org/10.1254/jphs.10240FP]

Keywords: uromodulin, androgen, urate transport system, transgenic mice, kidney

\section{Introduction}

Uromodulin storage diseases are rare autosomal dominant disorders characterized by hyperuricemia of the underexcretion type, gout, and renal insufficiency (1). These diseases are caused by mutations in the UMOD gene that encodes uromodulin (2). Uromodulin, known as Tamm-Horsfall protein, is the most abundant glycoprotein in normal human urine and distributed mainly in the epithelial cells of the thick ascending limb (TAL) of Henle's loop $(3,4)$. Although the physiological roles of uromodulin still remain elusive, it is suggested that uromodulin is associated with urine concentration ability and $\mathrm{NaCl}$ transport using the uromodulin-knockout mouse approach (5). Moreover, uromodulin is exported from the endoplasmic reticulum (ER) to the plasma membrane, and then released into the urine. On the other

*Corresponding author. hosoyamada-mk@pha.keio.ac.jp Published online in J-STAGE on February 24, 2011 (in advance) doi: 10.1254/jphs. 10240FP hand, it has been reported that mutations in the UMOD gene result in the accumulation of uromodulin in the TAL cells of uromodulin storage diseases because its transport is delayed (6). However, the effect of uromodulin mutation on the kidney has not been clearly established.

We previously reported the effect of mutant human uromodulin on the kidney by comparing transgenic mice harboring the $\mathrm{C} 148 \mathrm{~W}$ mutant human UMOD gene (UMOD-C148W Tg mice) and control C57/BL6 mice (7). However, the investigation required mice expressing the human wild-type isoform to evaluate the specific effect of uromodulin mutation on the kidney. In this study, we produced transgenic mice harboring the wild-type human UMOD gene (UMOD-WT Tg mice) to use as controls against UMOD-C148W Tg mice to demonstrate the effect of human uromodulin, with and without mutation, on the kidney. 


\section{Materials and Methods}

\section{Animals}

The DNA fragment of mouse UMOD promoter was ligated upstream into human UMOD cDNA containing pcDNA3.1(+) vector (Fig. 1A) as described previously (7). The transgene was microinjected into fertilized eggs of C57/BL6 inbred mice for UMOD-WT Tg mice production by the PhoenixBio Co., Ltd. (Tochigi). UMODWT Tg mice were genotyped as described previously (7). Twenty-four week-old male mice were anesthetized by intra-peritoneal injections of $50 \mathrm{mg} / \mathrm{kg}$ pentobarbital in order to obtain samples of plasma and kidney. Plasma and urinary urate and plasma allantoin concentrations were determined with HPLC (8). All animal experiments were carried out in accordance with the Keio University Guide for the Care and Use of Laboratory Animals.

\section{Immunofluorescence}

The frozen sections prepared from the kidneys of UMOD-WT Tg mice were double-stained with antihuman and anti-mouse uromodulin antibodies (Santa Cruz Biotechnology, Inc., Santa Cruz, CA, USA) using the Qdot $^{\circledR}$ nanocrystal technology (Life Technologies Japan, Ltd., Tokyo) (7).

\section{Microarray analysis}

Total RNA was extracted from the kidneys of UMODC148W and UMOD-WT Tg mice and purified as described previously (7). Microarray analysis was performed using Affymetrix Mouse Gene ST 1.0 arrays and analyzed using Expression Console by the PharmaFrontier Co., Ltd. (Kyoto). Renal-enriched genes were identified based on a 1.5 -fold enhancement of expression in UMOD-C148W versus UMOD-WT Tg mice, except that the signal strength of UMOD-WT Tg mice was less than 100 .

\section{Real-time PCR}

Total RNA was reverse-transcribed and amplified (8). The real-time PCR analyses used the TaqMan ${ }^{\circledR}$ probes corresponding to androgen related genes such as 5 - $\alpha$-reductase 2 (Srd5a2), $\beta$-glucuronidase (Gusb), ornithine decarboxylase structural 1 (Odc1), and cytochrome P450 (Cyp) 4a12a or urate transport systems-related genes such as urate transporter 1 (Urat1), glucose transporter 9 (Glut9), sodium-coupled monocarboxylate transporter 1 and $2($ Smct1/2) and ATP-binding cassette sub-family G member 2 (Abcg2), and 18s rRNA as an internal standard.

\section{Western blotting}

Microsomal fractions prepared as described previously

A

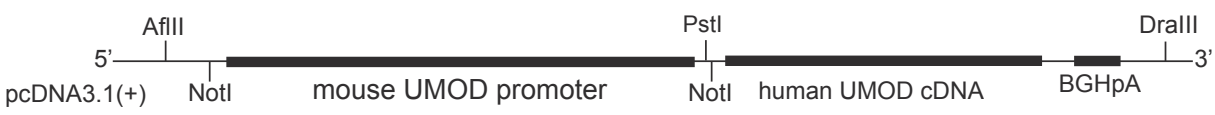

$\begin{array}{r}0.5 \mathrm{~kb} \\ \hline\end{array}$

B

UMOD-WT Tg

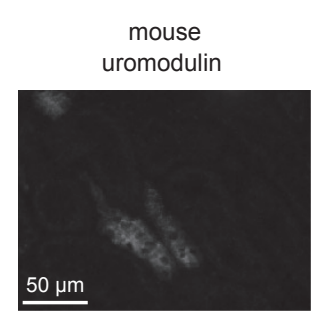

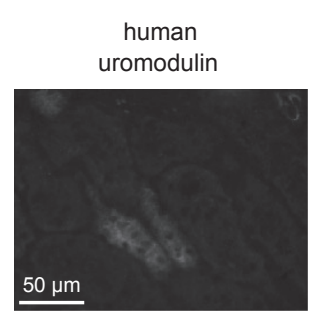

C

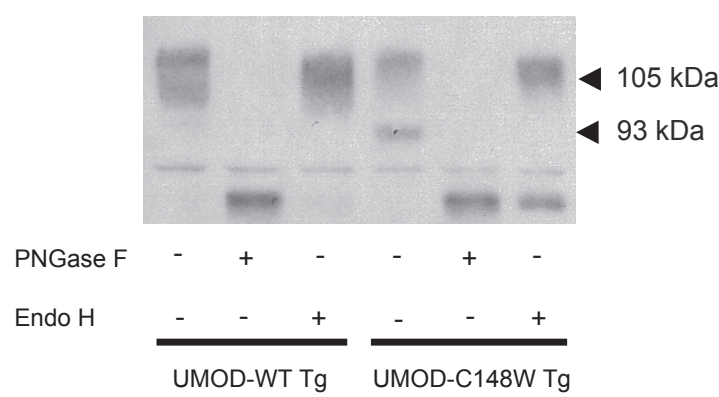

Fig. 1. Production and characterization of UMOD-WT Tg mice. A) Transgene of UMOD-WT Tg mice. The DNA fragment of mouse UMOD promoter was ligated upstream into human UMOD cDNA containing pcDNA3.1(+) vector. B) Expression of human uromodulin in the kidneys of UMOD-WT Tg mice. The frozen sections were double-stained with anti-human and anti-mouse uromodulin antibodies. Human and mouse uromodulin showed the same staining patterns. Magnification: $\times 400$. C) Deglycosylation of human uromodulin in the kidneys of UMOD-C148W and UMOD-WT Tg mice. Western blot analyses of the microsomal fractions of kidneys treated with PNGase F or Endo H. The 93-kDa human uromodulin was cleaved by Endo H. The 105-kDa human uromodulin was cleaved by PNGase F and Endo H. 
(7) were treated with peptide- $N$-glycosidase F (PNGase F) or endoglycosidase $\mathrm{H}$ (Endo $\mathrm{H}$ ) in accordance with the manufacturer's instructions (New England Biolabs Japan, Inc., Tokyo). The samples were separated by SDS-PAGE and transferred to a PVDF membrane. After blocking, the membrane was stained using anti-human uromodulin antibody $(1.0 \mu \mathrm{g} / \mathrm{mL}$; Santa Cruz Biotechnology, Inc.) or anti-Urat1 antibody $(0.21 \mu \mathrm{g} / \mathrm{mL})(8)$. Product detection was performed with an ECL kit (GE Healthcare UK Ltd., Little Chalfont, UK).

\section{Statistical analyses}

All data were expressed as the means \pm standard errors (S.E.M.). Differences between groups were analyzed statistically using one-way analysis of variance (ANOVA). Post hoc analysis was performed using Scheffe's test. $P$-values $<0.05$ were considered statistically significant.

\section{Results}

The breeding ability of UMOD-WT Tg mice was normal and one strain was selected based on mRNA level and immunofluorescence staining of human uromodulin in the kidney. UMOD-WT Tg mice were maintained by breeding male transgenic and female C57/BL6 mice, and heterozygous transgenic mice were used in this study. Moreover, immunofluorescence staining for human and mouse uromodulin in the kidneys of UMOD-WT Tg mice were performed to confirm the localization of human uromodulin. Human and mouse uromodulin were both detected in the same region (Fig. 1B). Therefore, the localization was similar to that of mutant human uro- modulin in the kidneys of UMOD-C148W Tg mice (7).

The microsomal fractions of the kidneys of UMODC148W and UMOD-WT Tg mice were treated with PNGase F or Endo H and subjected to western blot analyses to determine the characteristic of human uromodulin protein. Human uromodulin comprised of 93- and 105$\mathrm{kDa}$ fractions were expressed in the kidneys of UMODC148W Tg mice, while only the $105-\mathrm{kDa}$ component was observed in UMOD-WT Tg mice. Moreover, the 93-kDa human uromodulin was cleaved by PNGase F and Endo H. The 105-kDa component in both mice was not cleaved by Endo H (Fig. 1C). Therefore, Endo Hsensitive human uromodulin was expressed only in the kidneys of UMOD-C148W Tg mice.

We next measured the changes in gene expression of UMOD-C148W Tg mice using microarray technology. Out of 25,529 genes, 82 genes were induced and 79 genes were suppressed (Supplementary Table 1: available in the online version only). The genes that were identified based on a 2.5-fold enhancement of expression in UMOD-C148W versus UMOD-WT Tg mice were listed in Table 1. One of the strongly induced genes in UMODC148W Tg mice was Srd5a2, an enzyme that converts testosterone into the more potent androgen 5- $\alpha$ dihydrotestosterone ( $5 \alpha$-DHT). Quantitative real-time PCR confirmed the expressional changes in Srd5a2 seen in microarray analyses. The Srd5a2 mRNA level in UMOD-C148W Tg mice $(233.5 \pm 51.0 \%)$ was significantly higher than in UMOD-WT Tg mice $(66.0 \pm 25.5 \%$, $P<0.05$ ) (Fig. 2A). Moreover, quantitative real-time PCR was performed using the probes for androgen induced genes to evaluate the androgen action in the kidneys of UMOD-C148W Tg mice. The mRNA levels of

Table 1. Induced and suppressed genes in the kidneys of UMOD-C148W Tg mice

\begin{tabular}{llc}
\hline Gene symbol & Full name & $\begin{array}{c}\text { Fold change } \\
\text { (UMOD-C148W / UMOD-WT) }\end{array}$ \\
\hline Induced genes & & 3.15 \\
Ang & angiogenin, ribonuclease, RNase A family, 5 & 2.93 \\
Fbln7 & fibulin 7 & 2.92 \\
Srd5a2 & steroid 5 alpha-reductase 2 & 2.54 \\
Cc128 & chemokine (C-C motif) ligand 28 & 0.21 \\
Suppressed genes & 0.21 \\
Bc16 & B-cell leukemia/lymphoma 6 & 0.28 \\
Ces1 & carboxylesterase 1 & 0.31 \\
Slc7a12 & solute carrier family 7 (cationic amino acid transporter, y+ system), member 12 & 0.32 \\
EG665955 & endogenous virus Friend spleen focus-forming virus (SFFVp) cell-line DS19-sc9 & \\
Gfra1 & glial cell line derived neurotrophic factor family receptor alpha 1 & \\
\hline
\end{tabular}


Gusb, Odc1, and Cyp4a12a in UMOD-C148W Tg mice were significantly increased to $172.3 \pm 12.3 \%(P<0.01)$, $126.9 \pm 8.2 \%(P<0.01)$, and $122.3 \pm 8.8 \%(P<0.05)$ of those in UMOD-WT Tg mice $(95.4 \pm 10.5 \%, 87.4 \pm 5.4 \%$, and $78.8 \pm 8.2 \%$, respectively) (Fig. 2: B - D). Therefore, the androgen-related genes were increased in the kidneys of UMOD-C148W Tg mice.

In addition, quantitative real-time PCR was performed to assess the changes in the renal urate transport systems between UMOD-C148W and UMOD-WT Tg mice because hyperuricemia occurred frequently in uromodulin storage diseases and there are previous reports describing the regulation of urate transport systems by androgen treatment (8). Although there were no statistical differences in the mRNA levels of Glut9, Smct1/2, and Abcg2, the mRNA level of Urat1 in UMOD-C148W Tg mice was increased to $138.0 \pm 3.7 \%(P<0.05)$ of that in UMOD-WT Tg mice (120.1 $\pm 5.3 \%)$ (Fig. 3). However, there were no statistical differences in the protein level of Urat1. Also, plasma and urinary urate and plasma allantoin concentrations were not different between UMOD-C148W and UMOD-WT Tg mice (plasma urate:
$5.03 \pm 0.90$ and $5.63 \pm 1.16 \mu \mathrm{M}$, respectively; urinary urate: $215 \pm 12$ and $220 \pm 19 \mathrm{mmol} / \mathrm{mol} \mathrm{Cr}$, respectively; plasma allantoin: $38.32 \pm 7.07$ and $23.46 \pm 4.62 \mu \mathrm{M}$, respectively, $P=0.13$ ).

\section{Discussion}

We investigated the effect of uromodulin mutation on the intracellular localization of human uromodulin protein by comparing transgenic mice expressing human uromodulin with and without mutation. Two molecular weights of human uromodulin (93 and $105 \mathrm{kDa})$ were expressed in the kidneys of UMOD-C148W Tg mice and only the $93-\mathrm{kDa}$ component was cleaved by Endo $\mathrm{H}$. PNGase F cleaves all types of oligosaccharides of Nlinked glycoproteins from the ER and the Golgi apparatus, but Endo $\mathrm{H}$ cleaves the high mannose type of them from the ER. Choi et al. reported that the lower molecular weight uromodulin exists as a precursor form in the ER and the higher molecular weight component exists as a mature form in the Golgi apparatus and plasma membrane (9). Thus, the 93- and 105-kDa human uromodulins
A. Srd5a2

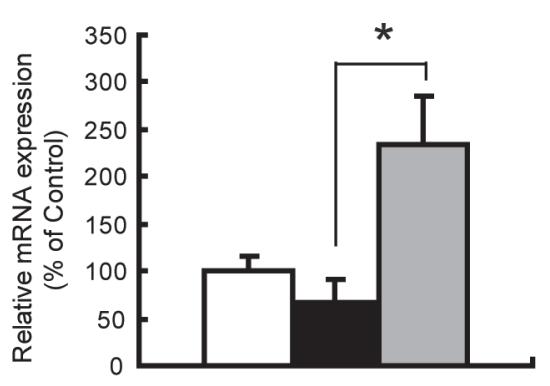

C. Odc1

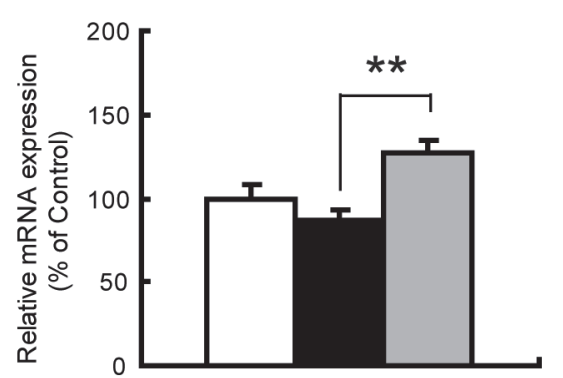

B. Gusb

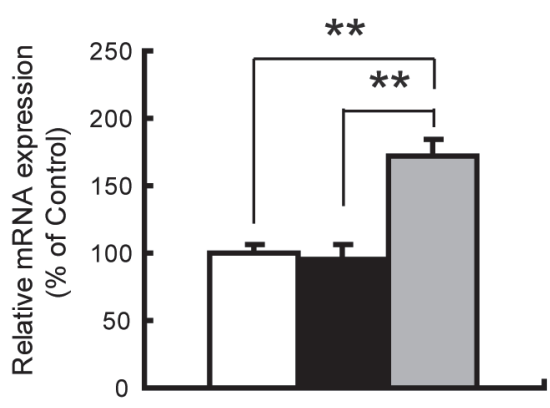

D. Cyp4a12a

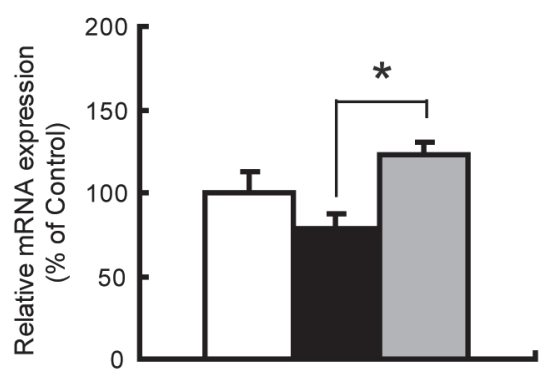

Fig. 2. Change of androgen related genes in the kidneys of UMOD-C148W Tg mice. Quantitative real-time PCR analyses were conducted using probes corresponding to Srd5a2 (A), Gusb (B), Odc1 (C), or Cyp4a12a (D) and 18s rRNA as an internal standard. The mRNA levels in control mice were fixed at $100 \%$. Data represent the mean \pm S.E.M., $\mathrm{n}=4-5, * P<0.05, * * P<0.01$. Renal mRNA levels of Srd5a2, Gusb, Odc1, and Cyp4a12a in UMOD-C148W Tg mice were higher than those in UMOD-WT Tg mice. 
A. Urat1

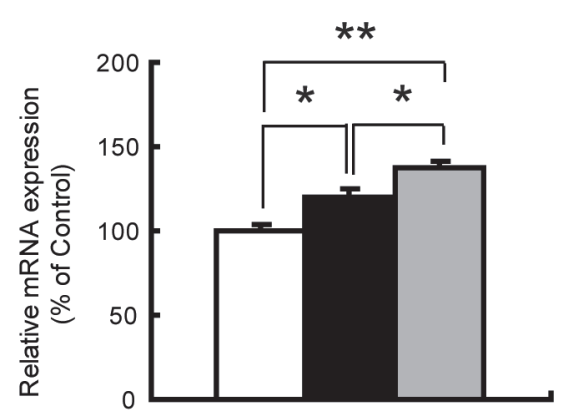

C. Smct1

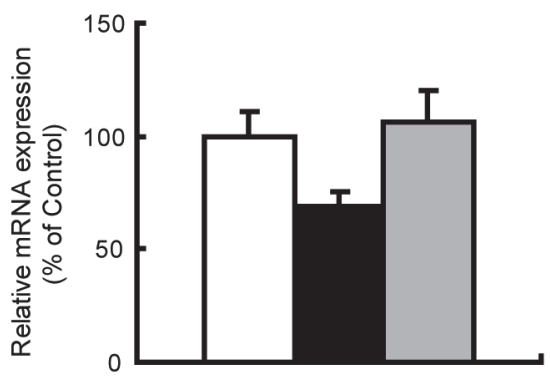

B. Glut9

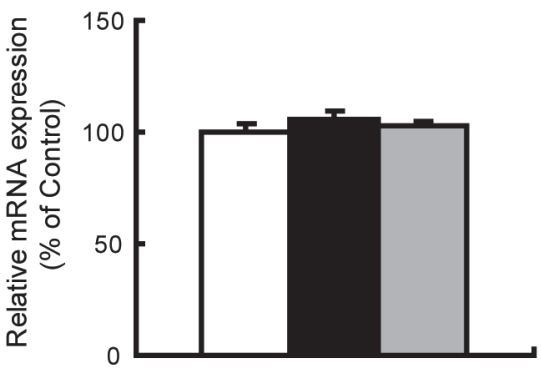

Control

- UMOD-WT Tg

口 UMOD-C148W Tg

Fig. 3. Urate transport systems in the kidneys of UMOD-C148W Tg mice. Quantitative real-time PCR analyses were conducted using probes corresponding to Urat1 (A), Glut9 (B), Smct1 (C), Smct2 (D), or Abcg2 (E) and 18s rRNA as an internal standard. The mRNA levels in control mice were fixed at $100 \%$. Data represent the mean \pm S.E.M., $\mathrm{n}=4-5, * P<0.05,{ }^{*} P<0.01$. Renal mRNA level of Urat1 in UMOD-C148W Tg mice was higher than that in UMOD-WT Tg mice.

may correspond to the precursor and mature forms, respectively. Our previous study reported that the $93-\mathrm{kDa}$ form was hardly expressed in the ER because the protein was mainly detected in the Triton-insoluble fraction (lipid raft) (7). However, there is a report that ER-resident proteins are detected in the Triton-insoluble membranes (10). Additionally, Bonnon et al. reported that a glycosylphosphatidylinositol (GPI)-anchored protein partitions into lipid-raft-like domains within the membranes of the ER (11). Consequently, the 93-kDa human uromodulin may be the precursor form in the ER because uromodulin is a GPI-anchored protein (12). This would concur with some reports that the $\mathrm{C} 148 \mathrm{~W}$ mutant uromodulin is retained in the ER (6) and ER-type uromodulin is increased in the kidneys of transgenic mice expressing the $\mathrm{C} 147 \mathrm{~W}$ mutant mouse uromodulin, which corresponds to the C148W mutant human uromodulin (13). Therefore, we consider that the uromodulin mutation affects the intracellular localization of human uromodulin, and UMODC148W Tg mice can serve as a model to study the effect of uromodulin mutation on the kidney.

Next, we investigated the effect by microarray tech- nology. Since the rate of formation of androgen depends on the level of expression of the specific androgen-synthesizing enzyme in the target tissue (14), we considered that renal $5 \alpha$-DHT level was increased by up-regulation of Srd5a2 mRNA level. Then, we confirmed the change in androgen induced genes in the kidney because it is reported that measurements of serum testosterone and $5 \alpha$-DHT level have little or no value except as an index of testicular activity since testosterone and $5 \alpha$-DHT are synthesized locally in the target tissue (15). As a result, the mRNA levels of androgen-induced genes (Gusb, Odc1, and Cyp4a12a) were increased in the kidneys of UMOD-C148W Tg mice. There is a report that the mRNA levels of Gusb and Odc were strongly induced in the kidneys of mice treated with androgens (16). Muller et al. reported that the treatment with $5 \alpha$-DHT significantly induced the mRNA level of Cyp4a12a, an enzyme that hydrolyzes arachidonic acid to 20-hydroxyeicosatetraenoic acid (20-HETE) in the mouse kidney (17). Moreover, Cyp4a12 and 20-HETE are associated with the renal epithelial cell proliferation in a mouse model of polycystic kidney disease (18), and a renal cyst is a clini- 
cal feature of uromodulin storage diseases (1). Although it represents the results of only one strain, the androgen action was increased in the kidneys of UMOD-C148W $\mathrm{Tg}$ mice. This would be related to a report that hyperuricemia was more frequent and severe in male patients and the progression of renal insufficiency tends to be worse in men than in women (19). Further studies are urgently needed to clarify the mechanism of enhancement involved in the androgen action, but we postulated as follows: First, the TAL-specific transporter sodium potassium chloride cotransporter 2 (NKCC2) is down-regulated like in the kidneys of transgenic mice expressing the mutant mouse uromodulin (13). Then, plasma angiotensin II level might be increased like in type I Batter's syndrome caused by a loss of function-type mutation in NKCC2 $(20,21)$. Finally, insulin-like growth factor I, induced by angiotensin II (22), might have increased 5 - $\alpha$-reductase activity (23).

We also investigated the effect of uromodulin mutation on the urate transport systems to evaluate the relationship between uromodulin mutation and hyperuricemia, which is the earliest onset of uromodulin storage diseases. Renal urate handling is maintained by the balance between reabsorption and secretion via several urate transporters. The transporters for regulation of serum urate levels are urate reabsorptive transporters, such as Urat1 and Glut9, and urate efflux transporter Abcg2 (24). Urat1 is a urate/anion exchanger and the activity of this protein is driven by cellular anions, which are reabsorbed via Smct1/2 (25). The mRNA level of Urat1 was increased in the kidneys of UMOD-C148W Tg mice. Urat1 mRNA level is enhanced by $5 \alpha$-DHT treatment (26). We recently reported that the mRNA levels of Urat1 and Smctl are increased by testosterone treatment, but those of Glut9 and Smct2 are decreased (8). Therefore, we suggest that androgen may affect transcriptional enhancement of Urat1 in the kidneys of UMOD-C148W Tg mice. However, there are the differences in mRNA expressions of Glut 9 and Smct1/2 in this study from our previous study. The change in mRNA level of Urat1 was shown by human uromodulin overexpression itself. The regulation of urate transport systems in this study may be attributed to factors other than androgen (27) or by the difference of concentrations between extrinsic and intrinsic androgen. Since the increase in mRNA level of the urate reabsorptive transport system was investigated, but the change in its protein level was not, we could not demonstrate the relationship between uromodulin mutation and renal urate handling until 24 weeks. The investigation requires the use of mice older than 24 weeks because it was shown that plasma allantoin concentration tends to be increased by uromodulin mutation in our experimental animals that metabolize urate to allantoin.
Further investigation is still needed using an androgenblocking agent such as flutamide (28) to confirm that androgen induced genes and urate reabsorptive transport systems were increased by the enhancement of androgen action via Srd5a2 up-regulation.

In conclusion, it is suggested that uromodulin mutation may be responsible for the enhancement of renal androgen action.

\section{Acknowledgments}

The authors thank Sanae Nakagomi and Nana Iwakawa for technical assistance. This study was supported in part by Grants-in-Aid from the Ministry of Education, Culture, Sports, Science, and Technology (Grant 20590547) and from the Gout Research Foundation.

\section{References}

1 Scolari F, Caridi G, Rampoldi L, Tardanico R, Izzi C, Pirulli D, et al. Uromodulin storage diseases: clinical aspects and mechanisms. Am J Kidney Dis. 2004;44:987-999.

2 Hart TC, Gorry MC, Hart PS, Woodard AS, Shihabi Z, Sandhu J, et al. Mutations of the UMOD gene are responsible for medullary cystic kidney disease 2 and familial juvenile hyperuricaemic nephropathy. J Med Genet. 2002;39:882-892.

3 Serafini-Cessi F, Malagolini N, Cavallone D. Tamm-Horsfall glycoprotein: biology and clinical relevance. Am J Kidney Dis. 2003;42:658-676.

4 Kokot F, Duława J. Tamm-Horsfall protein updated. Nephron. 2000;85:97-102.

5 Bachmann S, Mutig K, Bates J, Welker P, Geist B, Gross V, et al. Renal effects of Tamm-Horsfall protein (uromodulin) deficiency in mice. Am J Physiol Renal Physiol. 2005;288:F559-F567.

6 Rampoldi L, Caridi G, Santon D, Boaretto F, Bernascone I, Lamorte G, et al. Allelism of MCKD, FJHN and GCKD caused by impairment of uromodulin export dynamics. Hum Mol Genet. 2003;12:3369-3384.

7 Takiue Y, Hosoyamada M, Yokoo T, Kimura M, Shibasaki T. Progressive accumulation of intrinsic mouse uromodulin in the kidneys of transgenic mice harboring the mutant human uromodulin gene. Biol Pharm Bull. 2008;31:405-411.

8 Hosoyamada M, Takiue Y, Shibasaki T, Saito H. The effect of testosterone upon the urate reabsorptive transport system in mouse kidney. Nucleosides Nucleotides Nucleic Acids. 2010; 29:574-579.

9 Choi SW, Ryu OH, Choi SJ, Song IS, Bleyer AJ, Hart TC. Mutant Tamm-Horsfall glycoprotein accumulation in endoplasmic reticulum induces apoptosis reversed by colchicine and sodium 4-phenylbutyrate. J Am Soc Nephrol. 2005;16:3006-3014.

10 Sevlever D, Pickett S, Mann KJ, Sambamurti K, Medof ME, Rosenberry TL. Glycosylphosphatidylinositol-anchor intermediates associate with triton-insoluble membranes in subcellular compartments that include the endoplasmic reticulum. Biochem J. 1999;343:627-635.

11 Bonnon C, Wendeler MW, Paccaud JP, Hauri HP. Selective export of human GPI-anchored proteins from the endoplasmic reticulum. J Cell Sci. 2010;123:1705-1715.

12 Rindler MJ, Naik SS, Li N, Hoops TC, Peraldi MN. Uromodulin 
(Tamm-Horsfall glycoprotein/uromucoid) is a phosphatidylinositol-linked membrane protein. J Biol Chem. 1990;265:20784 20789.

13 Bernascone I, Janas S, Ikehata M, Trudu M, Corbelli A, Schaeffer $\mathrm{C}$, et al. A transgenic mouse model for uromodulin-associated kidney diseases shows specific tubulo-interstitial damage, urinary concentrating defect and renal failure. Hum Mol Genet. 2010; 19:2998-3010.

14 Labrie F, Bélanger A, Luu-The V, Labrie C, Simard J, Cusan L, et al. DHEA and the intracrine formation of androgens and estrogens in peripheral target tissues: its role during aging. Steroids. 1998;63:322-328.

15 Labrie F. Intracrinology. Mol Cell Endocrinol. 1991;78:C113C118.

16 Berger FG, Watson G. Androgen-regulated gene expression. Annu Rev Physiol. 1989;51:51-65.

17 Muller DN, Schmidt C, Barbosa-Sicard E, Wellner M, Gross V, Hercule $\mathrm{H}$, et al. Mouse Cyp4a isoforms: enzymatic properties, gender- and strain-specific expression, and role in renal 20-hydroxyeicosatetraenoic acid formation. Biochem J. 2007; 403:109-118.

18 Park F, Sweeney WE, Jia G, Roman RJ, Avner ED. 20-HETE mediates proliferation of renal epithelial cells in polycystic kidney disease. J Am Soc Nephrol. 2008;19:1929-1939.

19 Bleyer AJ, Woodard AS, Shihabi Z, Sandhu J, Zhu H, Satko SG, et al. Clinical characterization of a family with a mutation in the uromodulin (Tamm-Horsfall glycoprotein) gene. Kidney Int. 2003;64:36-42.
20 Moriwaki Y, Yamamoto T, Takahashi S, Tsutsumi Z, Hada T. An atypical case of primary renal tubular hypokalaemic metabolic alkalosis with chronic tophaceous gout. Clin Rheumatol. 2001; 20:372-375.

21 Ogawa T, Fujise Y, Shibata S, Abiru M, Takigami Y, Miura T, et al. Bartter's syndrome with gouty arthritis. Intern Med. 1992; 31:1120-1124.

22 Delafontaine P, Lou H. Angiotensin II regulates insulin-like growth factor I gene expression in vascular smooth muscle cells. J Biol Chem. 1993;268:16866-16870.

23 Horton R, Pasupuletti V, Antonipillai I. Androgen induction of steroid 5 alpha-reductase may be mediated via insulin-like growth factor-I. Endocrinology. 1993;133:447-451.

24 Riches PL, Wright AF, Ralston SH. Recent insights into the pathogenesis of hyperuricaemia and gout. Hum Mol Genet. 2009; 18:R177-R184.

25 Anzai N, Kanai Y, Endou H. New insights into renal transport of urate. Curr Opin Rheumatol. 2007;19:151-157.

26 Cheng X, Klaassen CD. Tissue distribution, ontogeny, and hormonal regulation of xenobiotic transporters in mouse kidneys. Drug Metab Dispos. 2009;37:2178-2185.

27 Anzai N, Kanai Y, Endou H. Organic anion transporter family: current knowledge. J Pharmacol Sci. 2006;100:411-426.

28 Malstrom SE, Tornavaca O, Meseguer A, Purchio AF, West DB. The characterization and hormonal regulation of kidney androgen-regulated protein (Kap)-luciferase transgenic mice. Toxicol Sci. 2004;79:266-277. 